doi: $10.15407 /$ ujpe61.05.0413

D. FISCALETTI, ${ }^{1,2}$ A. SORLI ${ }^{1,2}$

1 SpaceLife Institute

(San Lorenzo in Campo (PU), Italy; e-mail: spacelife.institute@gmail.com)

${ }^{2}$ Foundations of Physics Institute

(Idrija, Slovenia; e-mail: sorli.amrit@gmail.com)

\title{
ABOUT A THREE-DIMENSIONAL QUANTUM VACUUM AS THE ULTIMATE ORIGIN OF GRAVITY, ELECTROMAGNETIC FIELD, DARK ENERGY ... AND QUANTUM BEHAVIOR \\ PACS 04.20.-q, 04.50.Kd 04.60.-m
}

\begin{abstract}
A unification model where matter is a structure of energy of a three-dimensional quantum vacuum and diminishes its energy density is proposed. Mass and gravity are carried by variable energy density of the three-dimensional quantum vacuum. Electric field and magnetic field are carried by regions of polarized quantum vacuum generated by appropriate oscillations depending on fluctuations of the quantum vacuum energy density. The quantum behavior of matter derives directly from elementary energy fluctuations of the three-dimensional quantum vacuum. Dark energy is itself energy of the three-dimensional quantum vacuum.
\end{abstract}

Ke ywords: space-time, time, three-dimensional quantum vacuum, mass, gravity, electromagnetic field, quantum physics, dark energy.

\section{Introduction}

In special and general theories of relativity, the fundamental arena of the universe is a four-dimensional space-time, where time is considered to be the $4^{\text {th }}$ physical dimension of the universal space. Our research confirms that time is not a physical dimension of space, time is merely a mathematical parameter (numerical order) of changes running in the universal space, which itself originates from a primordial timeless three-dimensional (3D) quantum vacuum [1, 2].

The existence of the physical vacuum as the ground (lowest energy density) state of a collection of quantum fields, in which the processes have origin can be considered as the most important consequence of modern quantum field theories: the quantum electrodynamics, the Weinberg-Salam-Glashow theory of electroweak interactions, and the quantum chromodynamics of strong interactions. The physical vacuum can be regarded as a real quantum arena (a kind of quantum fluid) filling out the whole universal space and corresponding to the lowest energy state. The quantum vacuum can be seen as an ever-changing collection of virtual particles which are created alone (photons) or in massive particle-antiparticle pairs,

(C) D. FISCALETTI, A. SORLI, 2016 both of which are jumping in and out of the existence within constraints of the Heisenberg uncertainty principle [3]. The real particles such as electrons, positrons, photons, hadrons, etc., as well as all macroscopic bodies, are quantum wave-like excitations of this medium endowed with certain quantum numbers ensuring their relative stability. The particles are bubbles guided by ocean waves. In other words, the ocean waves represent pilot-waves guiding the bubbles. Waves may be created and destroyed, but the ocean is eternal [4].

In the picture of the physical vacuum, one can say that the physical space, in which elementary particles and stellar objects move, has origin in a fundamental quantum vacuum endowed with fundamental quantum fields that exhibit zero-point fluctuations everywhere in space, even in regions, which are devoid of matter and radiation. These zero-point fluctuations of the quantum fields, as well as other "vacuum phenomena" of quantum field theory, give rise to an enormous vacuum energy density.

The discovery of the dark energy in cosmology and the estimate of its relatively low value leads to the problem regarding the mechanism of radical reduction of the vacuum energy density with respect to the predictions of local quantum field theories. The 
dark energy is associated to the cosmological density

$\rho_{\Lambda}=\Lambda / 8 \pi G=7.02 \times 10^{-24} \mathrm{~g} / \mathrm{m}^{3}$

(where $\Lambda$ is the cosmological constant, and $G$ is Newton's universal gravitation constant) that is introduced in order to reproduce the phase of acceleration in the late universe. As a result of the dark energy, the universe expands exponentially rapidly on a timescale of the order of the cosmological time $t_{\Lambda}=1.46 \times 10^{18} \mathrm{~s}$ (which represents its de Sitter solution), leading to the phase of late inflation. The vacuum energy corresponds instead to the Planck density

$\rho_{p}=5.16 \times 10^{99} \frac{\mathrm{g}}{\mathrm{m}^{3}}$

and is responsible for the inflation in the late universe. As a result of the vacuum energy, the universe expands exponentially rapidly on a timescale of the order of the Planck time $t_{\Lambda}=5.39 \times 10^{-44} \mathrm{~s}$. The Planck density (2) differs by (1) about 122 orders of magnitude, thus giving rise to the so-called cosmological constant problem.

In order to solve the cosmological constant problem, various authors proposed explanations for the actual value (1), which invokes the fluctuations of the quantum vacuum. In particular, in [5-7], Santos showed that a curvature of space-time similar to the curvature associated with a dark energy is generated by quantum vacuum fluctuations on the basis of the equation

$\rho_{\mathrm{DE}} \cong 70 G \int_{0}^{\infty} C(s) s d s$,

which states that the possible value of "dark energy" density is the product of Newton's gravitational constant and the integral of the two-point correlation function of vacuum fluctuations defined by

$C\left(\left|\mathbf{r}_{1}-\mathbf{r}\right|\right)=\frac{1}{2}\langle v a c| \hat{\rho}\left(\mathbf{r}_{1}, t\right) \hat{\rho}\left(\mathbf{r}_{2}, t\right)+$

$+\hat{\rho}\left(\mathbf{r}_{2}, t\right) \hat{\rho}\left(\mathbf{r}_{1}, t\right)|v a c\rangle$,

$\hat{\rho}$ being an energy density operator such that its vacuum expectation is zero, while the vacuum expectation of the square of it is not zero. The relations between the metric coefficients and the matter stressenergy tensor are non-linear, and, as a consequence, the expectation of the metric turns out to be not the same as the metric of the expectation of the matter tensor. The difference between these two quantities gives rise to a contribution of the vacuum fluctuations mimicking the effect of Einstein's cosmological constant.

In analogous way, Fomin and Fomina [8] suggested that the dark energy is determined by the gravitational interaction of the quantum fluctuations of a discrete crystal-like vacuum consisting of massless and charge-neutral cells having the size of Planck length. These spatially closed massless "Planck cells" interact with neighbors through quadrupole forces of the type of van der Waals forces. As a result, the energy density of zero-point oscillations of the vacuum radically reduces from formally infinite (for local quantum field theories) to finite and even close to zero.

Finally, in the recent paper [9], Daywitt claims that even a more fundamental vacuum state, the Planck vacuum, exists, from which the ordinary quantum vacuum would emerge. This primordial Planck vacuum would consist of an omnipresent degenerate gas of negative-energy Planck particles characterized by the Planck mass, Planck length, and charge $e_{*}^{2}=\frac{e^{2}}{\alpha^{2}}$ (which is a manifestation of its polarizability), where $\alpha$ is the fine structure constant. In Daywitt's model, the granular structure of the Planck vacuum has the effect to produce a truncation of the frequency spectrum of the zero-point fields for the virtual particles and to lead to a finite energy density and a finite renormalization mass.

By following the philosophy that is at the basis of the approaches here mentioned, the authors of this paper have recently introduced a unifying approach, in which general relativity emerges as the hydrodynamic limit of some underlying theory of a more fundamental microscopic 3D quantum vacuum condensate endowed with elementary reduction-state (RS) processes of creation/annihilation of quanta (more precisely, of virtual pairs particles-antiparticles) [10, 11]. We can call this approach as a "model of the $3 \mathrm{D}$ quantum vacuum". In this article, our aim is to show in what sense this approach allows us to obtain a unifying treatment of different phenomena such as gravity and the electromagnetic field, as well as the quantum behavior of matter. This paper is structured in the following way. In Section 2, we will provide a treatment of gravitation, by showing that the real explanation for the dark energy lies in the fluctuations

ISSN 2071-0194. Ukr. J. Phys. 2016. Vol. 61, No. 5 
of the 3D quantum vacuum energy density and that the curvature of space-time associated with a dark energy density is obtained as a consequence of more fundamental changes of the $3 \mathrm{D}$ quantum vacuum energy density. In other words, it can be physically defined as the mathematical value of the 3D quantum vacuum energy density. In Section 3 , we will analyze the electromagnetic field as a consequence of more fundamental properties of the $3 \mathrm{D}$ quantum vacuum, and we will introduce a suggestive re-reading of the weak equivalence principle inside this scheme. In Section 4 , we will show in what sense our 3D quantum vacuum model allows us also to reproduce the quantum behavior of matter: the behavior of subatomic particles, as well as the standard equations of quantum mechanics. The non-locality of quantum systems emerges directly from the formalism of the 3D quantum vacuum defined by the RS processes.

\section{Gravity, Curvature}

of Space-Time, Dark Energy ...

and RS Processes: from the Postulates of the Three-Dimensional Quantum Vacuum Model to the Fundamental Results

Our model starts from the consideration of a 3D quantum vacuum condensate characterized by a quantized structure at the Planck scale.

By starting from the Planckian metric (the system of units introduced by Max Planck in 1899 [12] from dimensionality considerations by combining the three fundamental world constants, namely the speed of light $c$, the Planck constant $h$, and the Newton's universal gravitational constant $G$ ), an interesting way to define a quantum vacuum energy density emerges. In the outer intergalactic space, namely in the absence of material objects, the energy density of the 3D quantum vacuum is physically associated with the total average volumetric energy density, owed to all the frequency modes possible within the visible size of the universe, in other words assumes the maximum value given by the Planck energy density

$\rho_{\mathrm{pE}}=\frac{m_{p} c^{2}}{l_{p}^{3}}=4.641266 \times 10^{113} \frac{\mathrm{J}}{\mathrm{m}^{3}}$,

where $m_{p}$ is the Planck mass. The Planck energy density (5) can be considered as the ground state of the same physical flat-space background. The appearance of material objects and subatomic particles corresponds to changes of the quantum vacuum energy density. Thus, they can be considered as the excited states of the same physical flat-space background. The excited state of the $3 \mathrm{D}$ quantum vacuum corresponding to the appearance of a material particle of mass $m$ is defined by a quantum vacuum energy density (in the center of this particle) given by equation

$\rho=\rho_{\mathrm{pE}}-\frac{m c^{2}}{V}$,

where $V$ is the volume of the particle [10-11, 13]. Gravity is a phenomenon determined by the changes of the quantum vacuum energy density and thus by the excited states of the same flat-space background. The appearance of a material particle of mass $m$ corresponds to an excited state of the 3D flat-space background defined by a diminishing of the energy density (with respect to the ground state) given by the equation

$\rho_{\mathrm{pE}}-\rho \equiv \Delta \rho=\frac{m c^{2}}{V}$.

In other words, each material particle endowed with mass is produced by a change of the quantum vacuum energy density on the basis of the equation

$m=\frac{V \Delta \rho}{c^{2}}$.

Equation (8) implies that the property of mass derives from a change of the quantum vacuum energy density. Equation (8) indicates that the mass of a given massive body or particle is a result of the interaction of that body or particle with space. In particular, it corresponds to an opportune change of the quantum vacuum energy density, which can be considered as the fundamental property of the universal space. Each body or particle diminishes the quantum vacuum energy density. We measure this decrease of the quantum vacuum energy density as "inertial mass" and as "gravitational mass".

In view of the results obtained by Santos in the already mentioned papers [5-7], the quantized metric of the $3 \mathrm{D}$ quantum vacuum condensate in our approach is

$d \hat{s}^{2}=\hat{g}_{\mu \nu} d x^{\mu} d x^{\nu}$,

whose coefficients (in polar coordinates) are defined by the equations

$\hat{g}_{00}=-1+\hat{h}_{00}, \hat{g}_{11}=1+\hat{h}_{11}, \hat{g}_{11}=1+\hat{h}_{11}$,

$\hat{g}_{33}=r^{2} \sin ^{2} \vartheta\left(1+\hat{h}_{33}\right), \hat{g}_{\mu \nu}=\hat{h}_{\mu \nu}$ for $\mu \neq \nu$, 
where the multiplication of every term by the unit operator is implicit and, at the order $O\left(r^{2}\right)$, one has

$$
\begin{aligned}
\left\langle\hat{h}_{\mu \nu}\right\rangle & =0 \quad \text { except } \\
\left\langle\hat{h}_{00}\right\rangle & =\frac{8 \pi G}{3}\left(\frac{\Delta \rho_{\mathrm{qvE}}}{c^{2}}+\frac{35 G c^{2}}{2 \pi \hbar^{4} V}\left(\frac{V}{c^{2}} \Delta \rho_{\mathrm{qvE}}^{\mathrm{DE}}\right)^{6}\right) r^{2},(11) \\
\left\langle\hat{h}_{11}\right\rangle & =\frac{8 \pi G}{3}\left(-\frac{\Delta \rho_{\mathrm{qvE}}}{2 c^{2}}+\frac{35 G c^{2}}{2 \pi \hbar^{4} V}\left(\frac{V}{c^{2}} \Delta \rho_{\mathrm{qvE}}^{\mathrm{DE}}\right)^{6}\right) r^{2} .
\end{aligned}
$$

In Eqs. (11), $V$ is the volume of the object under consideration, $\Delta \rho_{\mathrm{qvE}}^{\mathrm{DE}}$ are opportune fluctuations of the quantum vacuum energy density, which determine the dark energy density on the basis of the relation

$\rho_{\mathrm{DE}} \cong \frac{35 G c^{2}}{2 \pi \hbar^{4} V}\left(\frac{V}{c^{2}} \Delta \rho_{\mathrm{qvE}}^{\mathrm{DE}}\right)^{6}$.

Here, therefore, the dark energy emerges as the energy itself of the 3D quantum vacuum, and the fluctuations of the quantum vacuum energy density play the same role of Santos' two-point correlation function. In fact, there is an equivalence between the fluctuations of the quantum vacuum energy density and the two-point correlation function: in our approach, the fluctuations of the $3 \mathrm{D}$ quantum vacuum energy density act as a two-point correlation function (4) on the basis of the relation

$$
\frac{c^{4}}{4 \pi \hbar^{4}}\left(\frac{V}{c^{2}} \Delta \rho_{\mathrm{qvE}}^{\mathrm{DE}}\right)^{6} \cong \int_{0}^{\infty} C(s) s d s .
$$

The quantized metric (9) is associated with an underlying microscopic geometry expressed by the equations

$\Delta x \geqslant \frac{\hbar}{2 \Delta p}+\frac{\Delta p}{2 \hbar}\left(2 \pi^{2} / 3\right)^{2 / 3} l^{2 / 3} l_{P}^{4 / 3}$

which is the uncertainty in the measure of a position,

$\Delta t \geqslant \frac{\hbar}{2 \Delta E}+\frac{\Delta E T_{0}^{2}}{2 \hbar}$,

which is the time uncertainty, and

$\Delta L \cong \frac{\left(2 \pi^{2} / 3\right)^{1 / 3} l^{1 / 3} l_{p}^{2 / 3} T_{0} E}{2 \hbar}$,

which indicates in what sense the curvature of a region of size $L$ can be related to the presence of energy and momentum in it. Equations (10)-(12) are derived from the quantum uncertainty principle [14] and from the hypotheses of space-time discreteness on the Planck scale by following Ng's treatment [15-18], in which the structure of the space-time foam can be inferred from the accuracy in the measurement of a distance $l$ - in a spherical geometry over the amount of time $T=2 l / c$ it takes light to cross the volume given by

$\delta l \geqslant\left(2 \pi^{2} / 3\right)^{1 / 3} l^{1 / 3} l_{P}^{2 / 3}$.

The quantized metric (9) allows the quantum Einstein equations

$\hat{G}_{\mu \nu}=\frac{8 \pi G}{c^{4}} \hat{T}$

(where the quantum Einstein tensor operator $\hat{G}_{\mu \nu}$ is expressed in terms of the operators $\hat{h}_{\mu \nu}$ ) to be obtained directly. This means that the curvature of the space-time characteristic of general relativity may be considered as a mathematical value, which emerges from the quantized metric (9) and thus from changes and fluctuations of the quantum vacuum energy density (on the basis of Eqs. (10) and (11)). In other words, one can say that changes and fluctuations of the quantum vacuum energy density, through the quantized metric (9) of the quantum vacuum condensate, whose coefficients are defined by Eqs. (10) and (11) (and whose underlying microscopic geometry is described by Eqs. (14)-(16)), can be considered the origin of the curvature of space-time characteristic of general relativity [10].

The other fundamental point of our approach is that also the behavior of subatomic particles derives from the 3D quantum vacuum condensate defined by the quantized metric (9) (whose coefficients are directly associated with fluctuations of the quantum vacuum energy density on the basis of Eqs. (10) and (11)). In fact, in our model, in analogy with Chiatti's and Licata's transactional approach [19, 20], the elementary fluctuations of the quantum vacuum energy density physically correspond to the events of preparation of the initial state (creation of a particle or object from the 3D quantum vacuum) and of detection of the final state (annihilation or destruction of a particle or object from the $3 \mathrm{D}$ quantum vacuum). The creation and the annihilation of an elementary quantum are two only primary extreme physical events of the $3 \mathrm{D}$ quantum vacuum, and each corresponds to a

ISSN 2071-0194. Ukr. J. Phys. 2016. Vol. 61, No. 5 
peculiar reduction of the state vector (which is constituted of interaction vertices, in which real elementary particles are created or destroyed). They can be also called "RS processes" where RS stands for the state reduction [11].

Moreover, the evolution of fluctuations of the 3D quantum vacuum energy density can be associated with appropriate waves of the vacuum corresponding to the wave function, which describes the amplitude of creation or destruction events. The wave function describing the occurrence of a creation/destruction event for a quantum particle $Q$ of mass (14) at a point event $x$ is represented by the probability amplitudes $\psi_{Q, i}(x)$ (for creation events) and $\phi_{Q, i}(x)$ (for destruction events) of a spinor $C=\left(\begin{array}{c}\psi_{Q, i} \\ \phi_{Q, i}\end{array}\right)$ at two components. It can be written as a spinor $C=\left(\begin{array}{l}\psi_{Q, i} \\ \phi_{Q, i}\end{array}\right)$, which satisfies a time-symmetric extension of the Klein-Gordon quantum relativistic equation

$\left(\begin{array}{cc}H & 0 \\ 0 & -H\end{array}\right) C=0$,

where $H=\left(-\hbar^{2} \partial^{\mu} \partial_{\mu}+m^{2} c^{2}\right)$. Equation (19) corresponds to the equations

$\left(-\hbar^{2} \partial^{\mu} \partial_{\mu}+m^{2} c^{2}\right) \psi_{Q, i}(x)=0$

for creation events and

$\left(\hbar^{2} \partial^{\mu} \partial_{\mu}-m^{2} c^{2}\right) \phi_{Q, i}(x)=0$

for destruction events, which can also be conveniently written as

$\left(-\hbar^{2} \partial^{\mu} \partial_{\mu}+\frac{V^{2}}{c^{2}}\left(\Delta \rho_{\mathrm{qvE}}\right)^{2}\right) \psi_{Q, i}(x)=0$

for creation events and

$\left(\hbar^{2} \partial^{\mu} \partial_{\mu}-\frac{V^{2}}{c^{2}}\left(\Delta \rho_{\mathrm{qvE}}\right)^{2}\right) \phi_{Q, i}(x)=0$

for destruction events, respectively, where $m=$ $\frac{V \Delta \rho_{\mathrm{qvE}}}{c^{2}}(8)$ is the mass of a quantum particle under consideration. In the non-relativistic limit, Eq. (19) becomes a pair of the Schrödinger equations

$-\frac{\hbar^{2}}{2 m} \nabla^{2} \psi_{Q, i}(x)=i \hbar \frac{\partial}{\partial t} \psi_{Q, i}(x)$

for creation events and

$-\frac{\hbar^{2}}{2 m} \nabla^{2} \phi_{Q, i}(x)=-i \hbar \frac{\partial}{\partial t} \phi_{Q, i}^{*}(x)$

ISSN 2071-0194. Ukr. J. Phys. 2016. Vol. 61, No. 5 for destruction events, which read, respectively,

$$
\begin{aligned}
-\frac{\hbar^{2} c^{2}}{2 V \Delta \rho_{\mathrm{qvE}}} \nabla^{2} \psi_{Q, i}(x) & =i \hbar \frac{\partial}{\partial t} \psi_{Q, i}(x), \\
-\frac{\hbar^{2} c^{2}}{2 V \Delta \rho_{\mathrm{qvE}}} \nabla^{2} \phi_{Q, i}(x) & =i \hbar \frac{\partial}{\partial t} \phi_{Q, i}^{*}(x) .
\end{aligned}
$$

The creation of a quantum particle $Q$ of mass (8) is associated with the initial condition for $\psi_{Q, i}(x)$ in Eq. (26), which has only retarded solutions. This classically corresponds to a material point with impulse $\mathbf{p}$ and kinetic energy $E=\mathbf{p} \cdot \mathbf{p} / 2 m>0$. The destruction of a quantum particle $Q$ is associated with the "initial", actually the final, condition for $\psi_{Q, i}^{*}(x)$ in equation (27), which has only advanced solutions corresponding to a material point with kinetic energy $E=-\mathbf{p} \cdot \mathbf{p} / 2 m<0$. In general, however, two conditions are different and, therefore, generate different solutions for the two equations, which are not necessarily mutual complex conjugates.

By writing the two components of the spinor in polar form

$\psi_{Q, i}=\left|\psi_{Q, i}\right| \exp \left(\frac{i S_{Q, i}^{\psi}}{\hbar}\right)$,
$\phi_{Q, i}=\left|\phi_{Q, i}\right| \exp \left(\frac{i S_{Q, i}^{\phi}}{\hbar}\right)$,

and decomposing the real and imaginary parts of the Klein-Gordon equation (19), one obtains a couple of quantum Hamilton-Jacobi equations for the real part that, by imposing the requirement that they are Poincarè-invariant and have the correct nonrelativistic limit, assume the form

$\partial_{\mu}\left(\begin{array}{c}S_{Q, i}^{\psi} \\ S_{Q, i}^{\phi}\end{array}\right) \partial^{\mu}\left(\begin{array}{c}S_{Q, i}^{\psi} \\ S_{Q, i}^{\phi}\end{array}\right)=\frac{V^{2}}{c^{2}}\left(\Delta \rho_{\mathrm{qvE}}\right)^{2} \exp \left(\begin{array}{c}Q_{Q, i}^{\psi} \\ -Q_{Q, i}^{\phi}\end{array}\right)$

while the imaginary part gives the continuity equation

$\partial_{\mu}\left(\rho \partial^{\mu}\left(\begin{array}{c}S_{Q, i}^{\psi} \\ S_{Q, i}^{\phi}\end{array}\right)\right)=0$

where $\rho$ is the ensemble of particles associated with the spinor under consideration and

$Q_{Q, i}=\frac{\hbar^{2} c^{2}}{V^{2}\left(\Delta \rho_{\mathrm{qVE}}\right)^{2}}\left(\begin{array}{c}\frac{\left(\nabla^{2}-\frac{1}{c^{2}} \frac{\partial^{2}}{\partial t^{2}}\right)\left|\psi_{Q, i}\right|}{\left|\psi_{Q, i}\right|} \\ -\frac{\left(\nabla^{2}-\frac{1}{c^{2}} \frac{\partial^{2}}{\partial t^{2}}\right)\left|\phi_{Q, i}\right|}{\left|\phi_{Q, i}\right|}\end{array}\right)$ 
is the quantum potential of the vacuum. In the nonrelativistic limit, Eq. (30) becomes

$$
\begin{aligned}
& \frac{c^{2}}{2 V\left(\Delta \rho_{\mathrm{qvE}}\right)}\left(\begin{array}{l}
\left|\nabla S_{Q, i}^{\psi}\right|^{2} \\
\left.\nabla S_{Q, i}^{\phi}\right|^{2}
\end{array}\right)+ \\
& +Q_{Q, i}+\left(\begin{array}{c}
V \\
-V
\end{array}\right)=-\frac{\partial}{\partial t}\left(\begin{array}{c}
S_{Q, i}^{\psi} \\
S_{Q, i}^{\phi}
\end{array}\right),
\end{aligned}
$$

and Eq. (28) becomes

$$
\frac{\partial}{\partial t}\left(\begin{array}{c}
\left|\psi_{Q, i}\right|^{2} \\
\left|\phi_{Q, i}\right|^{2}
\end{array}\right)+\nabla\left(\begin{array}{l}
\frac{\left|\psi_{Q, i}\right|^{2} \nabla S_{Q, i}^{\psi}}{m} \\
\frac{\left|\phi_{Q, i}\right|^{2} \nabla S_{Q, i}^{\phi}}{m}
\end{array}\right)=0,
$$

where

$$
Q_{Q, i}=-\frac{\hbar^{2} c}{2 V\left(\Delta \rho_{\mathrm{qvE}}\right)}\left(\begin{array}{c}
\frac{\nabla^{2}\left|\psi_{Q, i}\right|}{\left|\psi_{Q, i}\right|} \\
\frac{\nabla^{2}\left|\phi_{Q, i}\right|}{\left|\phi_{Q, i}\right|}
\end{array}\right)
$$

is the non-relativistic quantum potential of the vacuum.

On the basis of Eqs. (30), (32), (33), and (35), one deduces therefore that the waves of the vacuum act in a non-local way through an appropriate quantum potential of the vacuum (which, so to speak, guides the occurring of the processes of creation or annihilation in the $3 \mathrm{D}$ quantum vacuum). The quantum potential of the vacuum can be seen as the primary mathematical reality, which emerges from the very real extreme primary physical realities, namely from the processes of creation and annihilation of elementary quanta. The quantum potential of the vacuum makes the 3D quantum vacuum a fundamentally nonlocal manifold. Equations (32) and (35) mean that, both in the relativistic and non-relativistic domains, the quantum potential - both regarding the processes of creation and regarding the processes of annihilation - has a non-local instantaneous action. In sum, the non-local connection between RS processes derives from the instantaneous action of the quantum potential guiding the evolution of the occurring of the processes of creation or annihilation of quanta (corresponding to opportune changes of the quantum vacuum energy density) in the different regions of the $3 \mathrm{D}$ quantum vacuum. The first and second components of the quantum potential regard, respectively, the processes of creation and annihilation of quanta in the $3 \mathrm{D}$ quantum vacuum.

\section{8}

In virtue of the primary physical reality of the processes of creation and annihilation and of the nonlocal action of the quantum potential, which is associated with the amplitudes of them (as well as of the opposed sign of its second component with respect to the first component, which seems to indicate that it is not possible to go backward in the physical time [11]), the duration of the processes in the $3 \mathrm{D}$ quantum vacuum from the creation of a particle or object till its annihilation has not a primary physical reality, but exists only in the sense of numerical order. In other words, the 3D quantum vacuum, as a fundamental medium subtending the observable forms of matter, energy, and space-time, is a timeless background. The behavior of the matter in the universe can be seen as an undivided network of RS processes that take place in the $3 \mathrm{D}$ timeless quantum vacuum, and time exists merely as a mathematical parameter measuring the dynamics of a particle or object.

Let us see, then, to synthesize the fundamental results of our model about gravity, curvature of spacetime, dark energy, and reduction-state processes of quanta. In the light of what we have seen in this section, the foundational ideas of our model can be embedded in the following postulates (which can be considered as the fundamental postulates of our 3D quantum vacuum energy density model):

1. The medium of space is an isotropic granular 3D "quantum vacuum" constituted by energetic packets having the size of Planck's volume and whose most universal physical property is the energy density.

2 . In the free space, without the presence of massive particles, the quantum vacuum energy density is at its maximum and is given by relation (5) which defines the so-called "ground state" of the $3 \mathrm{D}$ quantum vacuum.

3. In the three-dimensional space, the appearance of matter derives from an opportune excited state of the $3 \mathrm{D}$ quantum vacuum defined by an opportune change of the quantum vacuum energy density and corresponding to specific RS processes of creation/annihilation of quanta. The excited state of the quantum vacuum corresponding to the appearance of a material particle of mass $m$ is defined (at the center of that particle) by the energy density (6) (and by a change of the energy density (7) with respect to the ground state), and its evolution is determined by opportune RS processes of creation/annihilation of quanta described by a wave function with two com-

ISSN 2071-0194. Ukr. J. Phys. 2016. Vol. 61, No. 5 
ponents satisfying a time-symmetric extension of the Klein-Gordon quantum relativistic equation (19).

These three postulates here enunciated allow us, on one hand, to obtain, in the light of Santos' results, a curvature of space-time similar to the curvature produced by the "dark energy" density as a phenomenon associated with the quantized metric (9) of the 3D quantum vacuum condensate, whose coefficients are defined by Eqs. (10) and (11) (and whose underlying microscopic geometry is described by Eqs. (14)(16)). On the other hand, they allow us to derive the quantum behavior of subatomic particles in a nonlocal picture ruled by a quantum potential of the vacuum, which guides the occurring of the processes of creation or annihilation in the $3 \mathrm{D}$ quantum vacuum on the basis of Eqs. (30), (32), (33), and (35).

\section{The Electromagnetic Effects of the Three-Dimensional Quantum \\ Vacuum ... and the Equivalence Principle between Inertial Mass and Gravitational Mass}

In a series of recent papers [21-24], Sbitnev introduced the concept of a physical vacuum containing pairs of particles-antiparticles, which make up a Bose-Einstein condensate, thus creating a superfluid medium. Such a scenario is not unusual in physical systems. For example, at the transition of helium to the superfluid phase, coherent Cooper pairs of electrons arise through the exchange by phonons [25]. This attraction is due to the electron-phonon interaction. The phonons are thermal excitations of a lattice and, indeed, play the role of a background medium.

In our model, on the basis of postulate 3 , we can consider the $3 \mathrm{D}$ quantum vacuum as a superfluid medium, which consists of an enormous amount of RS processes of creation/annihilation of particles-antiparticles with opposite orientations of spins (namely these pairs possess zero spin and constitute an organized Bose ensemble). In this way, the $3 \mathrm{D}$ quantum vacuum can be characterized by the Einstein energy-momentum tensor

$T^{\mu \nu}=(\varepsilon+p) u^{\mu} u^{\nu}+p \eta^{\mu \nu}$.

In Eq. (36), $\varepsilon$ and $p$ are functions per unit volume expressed in units of pressure, and the metric tensor $\eta^{\mu \nu}$ has the spacelike signature $(-,+,+,+)$. From the energy-momentum tensor (36), one obtains the conservation law

$\partial_{\mu}\left(V T^{\mu \nu} / n\right)=0$,

where $n$ is the number of the RS processes of virtual subparticles characterizing the vacuum medium.

Now, by following the philosophy that underlines Sbitnev's hydrodynamic picture provided in [22], one obtains the first Fick's law in the relativistic limit from Eqs. (36)-(37) in the form

$j_{\mu}=-\frac{D}{c^{2}} \partial_{\mu}\left(\Delta \rho_{\mathrm{qvE}}\right)$,

where $D$ is the diffusion coefficient having the dimension of length ${ }^{2} /$ time. On the basis of relation (38), the diffusion flux vector can be seen as a result of the scattering of the subparticles of the RS processes characterizing the vacuum on one another. In particular, it turns out to be proportional to the negative value of the gradient of fluctuations of the quantum vacuum energy density. Hence, by assuming that, as a consequence of the motion of the virtual particles, space-time is filled with virtual radiation with frequency $\omega$, one may define

$D=\frac{c^{2}}{\omega}$.

Thus, taking into account that the zero-point of the radiation is $E=\frac{\hbar \omega}{2}$, one has

$D=\frac{\hbar c^{2} n}{2 \Delta \rho_{\mathrm{qvE}} V}$.

Equation (40) says that the diffusion coefficient associated with the scattering of the subparticles of the $\mathrm{RS}$ processes characterizing the vacuum on one another depends of the number of the RS processes of virtual subparticles characterizing the volume $V$ of the vacuum medium under consideration and of the corresponding fluctuations of the quantum vacuum energy density.

Moreover, as regards the motions of virtual particles originating from the RS processes of creation/annihilation of quanta, it is natural to assume that they have a weak dispersion around a certain average. One may suggest that the virtual particles on this level are characterized by collisions with one 
another, which are similar to the collisions of Brownian particles. Here, in particular, a little modification of Nelson's hypothesis about ether populated by Brownian subparticles can be considered [26]. In this picture, in contrast to the quasiclassical collisions of Brownian subparticles, the diffusion is achieved due to the uncertainty principle - the collisions induce the uncertainty of momenta of the scattered subparticles [27].

On the basis of Eqs. (39) and (40), the virtual radiation produced by the evolution of the RS processes has frequency

$\omega=\frac{2 \Delta \rho_{\mathrm{qvE}} V}{\hbar n}$.

Frequency (41) may be considered the origin of electromagnetic effects of the $3 \mathrm{D}$ quantum vacuum. The electromagnetic field inside a perfectly reflecting cavity can be seen as an expansion of infinite different modes of the fundamental 3D quantum vacuum, where each mode corresponds to an independent oscillation defined by frequency (41) produced by a specific RS process of creation/annihilation of quanta in correspondence to elementary fluctuations of the $3 \mathrm{D}$ quantum vacuum. This means that the spectral energy density for zero-point fluctuations characterizing the electromagnetic properties of the quantum vacuum is

$\rho\left(\Delta \rho_{\mathrm{qvE}}\right)=\frac{4\left(\Delta \rho_{\mathrm{qvE}}\right)^{3} V^{3}}{\hbar^{2} \pi^{2} n^{3} c^{3}}$.

By starting from Eqs. (41) and (42), the electric and magnetic properties of the quantum vacuum, and thus the electromagnetic field of space, may be directly derived, on one hand, by considering the semiclassical approach known as stochastic electrodynamics (SED) and, on the other hand, inside quantum electrodynamics (QED). According to the authors, both SED and QED may be coherently used in these computations regarding the electromagnetic zero-point fluctuations of the $3 \mathrm{D}$ quantum vacuum in the light of the fact that they proved to lead to concurrent results in the context of another model of a quantum vacuum proposed by Haisch, Rueda, Puthoff, Dobyns, and Sunahata in [28-34].

Let us consider, before all, SED. In this picture, the quantum fluctuations of the electric and magnetic fields can be seen as random plane waves summed over all possible modes, with each mode having the zero-point energy $\hbar \omega / 2$. The electric and magnetic fields deriving from the zero-point fluctuations of the $3 \mathrm{D}$ quantum vacuum can be written in the SED approximation as

$$
\begin{aligned}
& \mathbf{E}_{\tau}^{\mathrm{zp}}(\mathbf{r}, t)=\sum_{\lambda=1}^{2} \int d^{3} k\left(\Delta \rho_{\mathrm{qvE}} V / n \pi^{2}\right)^{1 / 2} \hat{\varepsilon}(\mathbf{k}, \lambda) \times \\
& \times \cos \left[\mathbf{k} \cdot \mathbf{r}-\frac{2 \Delta \rho_{\mathrm{qvE}} V}{\hbar n} t-\theta(\mathbf{k}, \lambda)\right], \\
& \mathbf{B}^{\mathrm{zp}}(\mathbf{r}, t)=\sum_{\lambda=1}^{2} \int d^{3} k\left(\Delta \rho_{\mathrm{qvE}} V / n \pi^{2}\right)^{1 / 2} \times \\
& \times[\hat{k} \times \hat{\varepsilon}(\mathbf{k}, \lambda)] \cos \left[\mathbf{k} \cdot \mathbf{r}-\frac{2 \Delta \rho_{\mathrm{qvE}} V}{\hbar n} t-\theta(\mathbf{k}, \lambda)\right] .
\end{aligned}
$$

According to relations (43) and (44), the electromagnetic radiation can be expressed as the expansion in plane waves, where the sum is over two polarization states, $\hat{\varepsilon}$ is a unit vector, $\mathbf{k}$ is the polarization vector such that $|\mathbf{k}|=\omega / c$ and $\theta(\mathbf{k}, \lambda)$ is a random variable uniformly distributed in the interval $(0,2 \pi)$ and independently for each wave vector $\mathbf{k}$ and polarization index $\lambda$. Now, by considering the transformation from a stationary frame to a Rindler frame (characterized by a constant proper acceleration), which experiences an asymmetric event horizon leading to a non-zero electromagnetic energy and a non-zero momentum flux. It is defined by the following velocity and Lorentz factor, respectively:

$$
\begin{aligned}
& \frac{v}{c}=\tanh \left(\frac{a \tau}{c}\right), \\
& \gamma_{\tau}=\cosh \left(\frac{a \tau}{c}\right),
\end{aligned}
$$

where $\alpha$ is object's proper acceleration, and $\tau$ its proper time, one obtains the following expressions for the electric and magnetic zero-point fluctuations:

$$
\begin{aligned}
& \mathbf{E}_{\tau}^{\mathrm{zp}}(0, \tau)=\sum_{\lambda=1}^{2} \int d^{3} k\left(\Delta \rho_{\mathrm{qvE}} V / n \pi^{2}\right)^{1 / 2} \times \\
& \times\left\{\hat{x} \hat{\varepsilon}_{x}+\hat{y} \cosh \left(\frac{a \tau}{c}\right)\left[\hat{\varepsilon}_{y}-\tanh \left(\frac{a \tau}{c}\right)(\hat{k} \times \hat{\varepsilon})_{z}\right]+\right. \\
& \left.+\hat{z} \cosh \left(\frac{a \tau}{c}\right)\left[\hat{\varepsilon}_{z}+\tanh \left(\frac{a \tau}{c}\right)(\hat{k} \times \hat{\varepsilon})_{y}\right]\right\} \times \\
& \times \cos \left[k_{x} \frac{c^{2}}{a} \cosh \left(\frac{a \tau}{c}\right)-\frac{2 \Delta \rho_{\mathrm{qvE}} c V}{a \hbar n} \sinh \left(\frac{a \tau}{c}\right)-\theta(\mathbf{k}, \lambda)\right],
\end{aligned}
$$

ISSN 2071-0194. Ukr. J. Phys. 2016. Vol. 61, No. 5 


$$
\begin{aligned}
& \mathbf{B}_{\tau}^{\mathrm{zp}}(0, \tau)=\sum_{\lambda=1}^{2} \int d^{3} k\left(\Delta \rho_{\mathrm{qvE}} V / n \pi^{2}\right)^{1 / 2} \times \\
& \times\left\{\hat{x}(\hat{k} \times \hat{\varepsilon})_{x}+\hat{y} \cosh \left(\frac{a \tau}{c}\right)\left[(\hat{k} \times \hat{\varepsilon})_{y}-\tanh \left(\frac{a \tau}{c}\right)(\hat{\varepsilon})_{z}\right]+\right. \\
& \left.+\hat{z} \cosh \left(\frac{a \tau}{c}\right)\left[\hat{\varepsilon}_{z}+\tanh \left(\frac{a \tau}{c}\right)(\hat{k} \times \hat{\varepsilon})_{y}\right]\right\} \times \\
& \times \cos \left[k_{x} \frac{c^{2}}{a} \cosh \left(\frac{a \tau}{c}\right)-\frac{2 \Delta \rho_{\mathrm{qvE}} c V}{a \hbar n} \sinh \left(\frac{a \tau}{c}\right)-\theta(\mathbf{k}, \lambda)\right],
\end{aligned}
$$

where $\hat{\varepsilon}_{x}$ is the scalar projection of the $\hat{\varepsilon}$ unit vector along the $x$-direction, and similarly for $\hat{\varepsilon}_{y}$ and $\hat{\varepsilon}_{z}$. Equations (43) and (44) (as well as Eqs. (47) and (48) for Rindler frames) indicate clearly that the electric and magnetic fields are two different kinds of polarization of the $3 \mathrm{D}$ quantum vacuum produced by the frequencies of the radiation associated with the motion of virtual particles of the RS processes, namely by the fluctuations of the quantum vacuum energy density.

Let us remember now that, in the approach proposed by Haisch, Rueda, and Puthoff in a series of papers [28-33], the inertial mass and the gravitational mass can be interpreted as effects of the electromagnetic quantum vacuum. In Haisch's, Rueda's, and Puthoff's approach, a change in the momentum of the radiation field associated with the electromagnetic zero-point energy instantaneously transiting through an object of a given volume (and interacting with the quarks and electrons in that object) creates the inertial mass of the object. In other words, inertia emerges as a kind of the acceleration-dependent electromagnetic quantum vacuum drag force acting upon electromagnetically interacting elementary particles (electrons and quarks). On the other hand, the gravitational mass - as manifest in weight - results from what may in a limited sense be viewed as the acceleration of the electromagnetic quantum vacuum past a fixed object.

As regards the model proposed by Haisch, Rueda, and Puthoff in [28], it must be emphasized, however, that the explanation of inertia as an "electromagnetic resistance arising from the known spectral distortion of the zero-point field in accelerated frames" has been criticized recently by Levin, who claims that this result contains errors due to incorrect physical and mathematical assumptions associated with taking a nonrelativistic approach in the calculation of the magnetic Lorentz force [35]. According to Levin's research, the force exerted on even a slow moving accelerated oscillator "remembers" the entire history of the accelerated motion including times, when its velocity could have any large value, and, moreover, the high-frequency approximation taken into consideration by the three authors is not justified.

In our approach, by considering the electric and magnetic properties of the quantum vacuum expressed by relations (43) and (44) (and, in the Rindler frame, by relations (47) and (48)), the explanation of the weak equivalence principle provided by Haisch, Rueda, and Puthoff may be derived, re-read, understood, and justified in a simple, suggestive, and unifying way. Our view suggests a picture where - contrary to Haisch's, Rueda's, and Puthoff's model, where there is an ad hoc high-frequency approximation - the frequencies defined by Eq. (41) represent the primary ontology. The mesoscopic level described by Haisch's, Rueda's, and Puthoff's model about the electromagnetic zero-point fluctuations of the vacuum as the origin of the inertial and gravitational mass can be seen here as a secondary ontological level, which emerges from the primary existence of an enormous amount of $\mathrm{RS}$ processes of the $3 \mathrm{D}$ quantum vacuum. These $\mathrm{RS}$ processes determine the creation/annihilation of virtual particles-antiparticles with opposite orientations of spins, whose motions generate a virtual radiation defined by specific frequencies (given by (41)). The mesoscopic level considered by Haisch's, Rueda's, and Puthoff's view can be obtained in our approach on the basis of the consideration that the frequencies of the electromagnetic zero-point fields analyzed by these three authors derive from more fundamental specific frequencies associated with the motions of virtual particles of the RS processes, and thus from the elementary energy fluctuations of the fundamental 3D quantum vacuum in agreement with the fundamental relation (41).

In our approach, the rate of change of the momentum, which acts on the electromagnetically interacting accelerating object, is determined by the oscillations of the radiation produced by the motions of virtual particles of the RS processes, and thus by the elementary fluctuations of the $3 \mathrm{D}$ quantum vacuum. It may be computed by observing that each individual inertial frame corresponds to its own (or proper) random zero-point fluctuations of the $3 \mathrm{D}$ quantum vacuum determining the electric and magnetic fields. For 
each inertial reference frame, its proper electromagnetic vacuum fluctuations are homogeneously and isotropically distributed. This means that the components of the net Poynting vector of the zero-point field of a given frame, when observed in that same frame, all should vanish.

Both the momentum density and the total momentum generated by the fluctuations of the 3D quantum vacuum inside the proper volume $V$ of the body exhibit a time rate of change and have a non-vanishing time derivative. Thus, although both the Poynting vector and the momentum determined by the fluctuations of the $3 \mathrm{D}$ quantum vacuum inside the body instantaneously vanish, their time derivatives at that coincidence time do not vanish. This creates a stress or tension in the $3 \mathrm{D}$ quantum vacuum which is manifested on the accelerated particle as the Rindler frame force.

By evaluating the Poynting vector for a uniform acceleration of the object under consideration in the $x$-direction, one finds

$\mathbf{N}^{\mathrm{zp}}(\tau)=-\hat{x} \frac{8 V^{3}}{3 \hbar^{2} \pi^{2} n^{3} c^{3}} \sinh \left(\frac{2 \alpha \tau}{c}\right) \int\left(\rho_{\mathrm{pE}}-\rho\right)^{3} d \rho$,

where $\boldsymbol{\alpha}=\alpha \hat{x}$ is the uniform proper acceleration of the object. As a consequence, the amount of radiative momentum carried by the radiations produced by the motions of the virtual particles of the RS processes - namely by the zero-point fluctuations of the $3 \mathrm{D}$ quantum vacuum - that are passing through and instantaneously contained in the accelerated object, is given by the relation

$\mathbf{p}^{\mathrm{zp}}(\tau)=-\hat{x} \frac{16 V^{4}}{3 \hbar^{2} \pi^{2} n^{3} c^{5}} v_{\tau} \gamma_{\tau} \int\left(\rho_{\mathrm{pE}}-\rho\right)^{3} d \rho$.

The interacting fraction of the zero-point momentum instantaneously passing through the object is therefore a fraction of (50), i.e.

$\mathbf{p}^{\mathrm{zp}}(\tau)=-\hat{x} \frac{16 V^{4}}{3 \hbar^{2} \pi^{2} n^{3} c^{5}} v_{\tau} \gamma_{\tau} \int \eta(\rho)\left(\rho_{\mathrm{pE}}-\rho\right)^{3} d \rho$,

where $\eta(\rho)$ is a fraction of the energy that interacts with the material particles contained in $V$. By taking the time derivative of (51) into account, one gets

$\frac{d \mathbf{p}^{\mathrm{zp}}}{d t}=\mathbf{f}^{\mathrm{zp}}=-\left[\frac{16 V^{4}}{3 \hbar^{2} \pi^{2} n^{3} c^{5}} \int \eta(\rho)\left(\rho_{\mathrm{pE}}-\rho\right)^{3} d \rho\right] \mathbf{a}$.
Equation (52) indicates that, in order to maintain the acceleration of such an object, a motive force $\mathbf{f}$ must continuously be applied to balance the counteracting reaction force produced by the fluctuations of the vacuum producing its electromagnetic properties (namely the Rindler frame force $\mathbf{f}^{\mathrm{zp}}$ ), which is given by

$\mathbf{f}=-\mathbf{f}^{\mathrm{zp}}=\left[4 \frac{V^{4}}{\hbar^{2} \pi^{2} n^{3} c^{5}} \int \eta(\rho)\left(\rho_{\mathrm{pE}}-\rho\right)^{3} d \rho\right] \mathbf{a}$,

where the factor $4 / 3$ has been suppressed in order to obtain the proper correct relativistic four-vector force expression. On the basis of Eq. (53), one can define the contribution to the inertial mass contained in $V$, due to the $3 \mathrm{D}$ quantum vacuum fluctuations, as

$m_{i}=\left[4 \frac{V^{4}}{\hbar^{2} \pi^{2} n^{3} c^{5}} \int \eta(\omega)\left(\rho_{\mathrm{pE}}-\rho\right)^{3} d \omega\right]$.

Thus, while in Haisch's and Rueda's quantum vacuum inertia hypothesis, some of the apparent inertial mass of an object originates in the interacting fraction of the zero-point energy instantaneously contained in an object with an electromagnetic quantum vacuum defined by fundamental zero-point electromagnetic vector fields, the electric and magnetic fields in our approach are not taken as fundamental, but have a secondary ontological status. In our approach, the inertial mass of the object emerges from the interacting fraction of an energy density characterizing electromagnetic properties of the 3D quantum vacuum, which are determined by the frequencies associated to opportune RS processes of creation/annihilation of quanta corresponding to elementary fluctuations of the quantum vacuum energy density. In this view, the apparent momentum of the object can be traced back to the momentum of the zero-point radiation field associated with the motion of virtual particles of the RS processes. The rest mass in Eq. (54) exactly corresponds to the amount of mass equivalent produced by the motions of virtual particles of the RS processes - associated with the elementary fluctuations of the quantum vacuum - enclosed within the volume of the body and that, thanks to the $\eta(\omega)$ spectral factor, interacts with the body. The function $\eta(\omega)$ physically measures the relative strength of the interaction between the oscillations associated with the radiation produced by the motions of virtual particles of the RS processes (corresponding to elementary fluctuations 
of the quantum vacuum energy density) and the massive object, whose interaction acts to oppose the acceleration. In analogy to Haisch's and Rueda's model, a stationary observer would come to the conclusion that the mass of an accelerating object is steadily increasing as $\gamma_{\tau} m$, but, in the accelerating frame, no mass change is evident. One's momentum in one's own reference frame is always zero. Physical consequences emerge only in correspondence to a change in the momentum generated by the oscillations of the radiation produced by the motions of virtual particles of the RS processes and, thus, by the elementary fluctuations of the $3 \mathrm{D}$ quantum vacuum.

Moreover, as regards the equivalence between inertial mass and gravitational mass, in our approach, the interaction between the fluctuations of the quantum vacuum originating its electromagnetic properties (as a consequence of the oscillations of the radiation field associated with the motion of the virtual particles of the RS processes) and the electromagneticallyinteracting particles constituting any physical object (quarks and electrons) is identical for the following two situations: acceleration with respect to constant velocity inertial frames or remaining fixed above some gravitating body with respect to freely falling local inertial frames. The existence of the Rindler frame force in an accelerating reference frame translate and correspond exactly to a reference frame fixed above a gravitating body. In the same manner that light rays are deviated from the straight-line propagation by a massive gravitating body, the other forms of electromagnetic radiation, including the electromagnetic zero-point field rays (in the SED approximation), are also deviated from the straight-line propagation.

In papers [30, 31], Rueda and Haisch interpreted the drag force exerted by the electromagnetic quantum vacuum radiation as the inertia reaction force of an object that is being forced to accelerate through the electromagnetic quantum vacuum field. But, in our approach, the drag force derives from the oscillations associated with the radiation produced by the motions of virtual particles of the RS processes, namely ultimately derives from elementary fluctuations of the energy density of the same 3D quantum vacuum. These fundamental zero-point fluctuations lead to the associated nonrelativistic form of the inertia reaction force

$\mathbf{f}_{*}^{\mathrm{zp}}=-m_{i} \mathbf{g}_{\omega}$, where $\mathbf{g}_{\omega}$ is the acceleration, with which the frequency $\omega$ appears in a local inertial frame $I_{*}$, and the coefficient $m_{i}$ is given by (54). Here, the coupling function $\eta(\omega)$ of the inertial mass (54) has different shapes for the electron, a given quark, a composite particle like the proton, a molecule, a homogeneous dust grain, or a homogeneous macroscopic body. However, what appears as inertial mass, $m_{i}$, to the observer in a local Lorentz frame $I_{*, L}$ is what corresponds to passive gravitational mass, $m_{g}$. Thus, one has

$m_{g}=\left[4 \frac{V^{4}}{\hbar^{2} \pi^{2} n^{3} c^{5}} \int \eta(\omega)\left(\rho_{\mathrm{pE}}-\rho\right)^{3} d \omega\right]$.

In synthesis, in our approach of the $3 \mathrm{D}$ quantum vacuum, the physical basis for the principle of equivalence is the fact that accelerating through the regions of quantum vacuum characterized by frequencies (41) of the radiation field determined by the motion of virtual particles of the RS processes is identical to remaining fixed in a gravitational field and having the electromagnetic properties of the $3 \mathrm{D}$ quantum vacuum fall past on curved geodesics. In both situations, the observer will experience an asymmetry in the radiation pattern of the electromagnetic properties of the quantum vacuum, which results in a force - either the inertia reaction force or weight - which becomes then the same thing within this more general Einsteinian perspective.

Let us analyze now the electromagnetic properties of the 3D quantum vacuum, by using the picture of QED. In this regard, let us remember that the significant results have been also obtained recently by Sunahata, Rueda, and Haisch in paper [34]. There, Sunahata, Rueda, and Haisch analyzed the interaction between the zero-point field and an object under hyperbolic motion (constant proper acceleration) within a formulation that uses the low-energy version of quantum electrodynamics, also called nonrelativistic quantum electrodynamics with the creation and annihilation operators for all the averaging calculations. In their treatment, Sunahata, Rueda, and Haisch found the appearance of a reaction force, which is proportional in magnitude, and opposite in direction, to the acceleration (and, thus, which can be interpreted as inertia). The three authors also pointed out that the equivalence principle between inertial mass and gravitational mass follows naturally in this picture and showed that QED leads to the same results re- 
ported in Rueda's and Haisch's papers [30-32] mentioned above, and, thus, that there is a contribution to the inertia reaction force coming from the electromagnetic quantum vacuum.

By using Sunahata's, Rueda's, and Haisch's formalism, let us consider an object, being at rest at time $t_{*}=0$ in the laboratory frame $I_{*}$, uniformly accelerated by an external force, which produces a rectilinear motion along the $x$-axis with constant proper acceleration $\mathbf{a}=a \hat{x}$. Consider the Rindler frame $S$ such that its $x$-axis coincides with that of $I_{*}$ and let the body be located at coordinates $\left(c^{2} / a, 0,0\right)$ in $S$ at all times. So, this point of $S$ performs hyperbolic motion. The acceleration of the body point in $I_{*}$ is $\mathbf{a}_{*}=\gamma_{\tau}^{-3} \mathbf{a}$ at the body proper time $\tau$. Consider also an infinite collection of inertial frames $\left\{I_{\tau}\right\}$ such that, at body's proper time $\tau$, the body is located at the point $\left(c^{2} / a, 0,0\right)$ of $I_{\tau}$. The $I_{\tau}$ frames have all axes parallel to those of $I_{*}$, and their $x$-axes coincide with that of $I_{*}$. By setting the proper time $\tau$ such that, at $\tau=0$, the corresponding $I_{\tau}$ coincides with $I_{*}$, one has $I_{\tau=0}=I_{*}$, and, thus, the hyperbolic motion assures that

$x_{*}=\frac{c^{2}}{a} \cosh \left(\frac{a \tau}{c}\right)$,

$t_{*}=\frac{c}{a} \sinh \left(\frac{a \tau}{c}\right)$,

$\beta_{\tau}=\frac{u_{x}(\tau)}{c}=\tanh \left(\frac{a \tau}{c}\right)$,

$\gamma_{\tau}=\left(1-\beta_{\tau}^{2}\right)^{-1 / 2}=\cosh \left(\frac{a \tau}{c}\right)$.

By using the Lorentz transformation from the laboratory frame $I_{*}$ into an instantaneously comoving frame $I_{\tau}$, the electromagnetic zero-point field vectors - deriving from the fluctuations of the $3 \mathrm{D}$ quantum vacuum $-\mathbf{E}_{\mathrm{zp}}$ and $\mathbf{B}_{\mathrm{zp}}$ of $I_{*}$, as represented in $I_{\tau}$, are given by the relations

$\mathbf{E}_{\tau}^{\mathrm{zp}}(0, \tau)=\sum_{\lambda=1}^{2} \int d^{3} k H_{\mathrm{zp}}(\omega)\left\{\hat{x} \hat{\varepsilon}_{x}+\hat{y} \cosh \left(\frac{a \tau}{c}\right) \times\right.$

$\times\left[\hat{\varepsilon}_{y}-\tanh \left(\frac{a \tau}{c}\right)(\hat{k} \times \hat{\varepsilon})_{z}\right]+\hat{z} \cosh \left(\frac{a \tau}{c}\right) \times$

$\left.\times\left[\hat{\varepsilon}_{z}+\tanh \left(\frac{a \tau}{c}\right)(\hat{k} \times \hat{\varepsilon})_{y}\right]\right\} \times$

$\times\left\{\boldsymbol{\alpha}(\mathbf{k}, \lambda) e^{i \theta}+\boldsymbol{\alpha}^{+}(\boldsymbol{k}, \lambda) e^{-i \theta}\right\}$,

$\mathbf{B}_{\tau}^{\mathrm{zp}}(0, \tau)=\sum_{\lambda=1}^{2} \int d^{3} k H_{\mathrm{zp}}(\omega)\left\{\hat{x}(\hat{k} \times \hat{\varepsilon})_{x}+\right.$

$$
\begin{aligned}
& +\hat{y} \cosh \left(\frac{a \tau}{c}\right)\left[(\hat{k} \times \hat{\varepsilon})_{y}-\tanh \left(\frac{a \tau}{c}\right)(\hat{\varepsilon})_{z}\right]+ \\
& \left.+\hat{z} \cosh \left(\frac{a \tau}{c}\right)\left[(\hat{k} \times \hat{\varepsilon})_{z}+\tanh \left(\frac{a \tau}{c}\right)(\hat{\varepsilon})_{y}\right]\right\} \times \\
& \times\left\{\boldsymbol{\alpha}(\mathbf{k}, \lambda) e^{i \theta}+\boldsymbol{\alpha}^{+}(\mathbf{k}, \lambda) e^{-i \theta}\right\} .
\end{aligned}
$$

In relations (61) and (62), we define

$\theta=\hat{k}_{x} \frac{c^{2}}{a} \cosh \left(\frac{a \tau}{c}\right)-\omega \frac{c}{a} \sinh \left(\frac{a \tau}{c}\right)$,

$\hat{\varepsilon}_{i}$ are the polarization components, $\mathbf{k}$ is the polarization wave vector, $\lambda$ is the polarization index, $\boldsymbol{\alpha}(\mathbf{k}, \lambda)$ and $\boldsymbol{\alpha}^{+}(\mathbf{k}, \lambda)$ are annihilation and creation operators, respectively. Moreover, by using QED, the spectral energy density for the zero-point fluctuations characterizing the electromagnetic properties of the quantum vacuum is $H_{\mathrm{zp}}^{2}(\omega)=\frac{\Delta \rho_{\mathrm{qvE}} V}{2 \pi^{2} n}$. Similarly to the results obtained in [34], the electric and magnetic fields associated with the fluctuations of the quantum vacuum, as seen in $I_{\tau}$, also correspond to the same fields as instantaneously seen in $S$ at the proper time $\tau$. By evaluating the Poynting vector determined by the fluctuations of the $3 \mathrm{D}$ quantum vacuum, which enters the volume of the accelerating object in the instantaneous co-moving frame $I_{\tau}$, at the $\left(c^{2} / a, 0,0\right)$ space point of the $I_{*}$ inertial frame, namely in $I_{\tau}$ at the $I_{\tau}$ space-time point,

$$
\begin{aligned}
& c t_{\tau}=-\frac{c^{2}}{a} \sinh \left(\frac{a \tau}{c}\right), \quad x_{\tau}=-\frac{c^{2}}{a} \cosh \left(\frac{a \tau}{c}\right), \\
& y_{\tau}=0, \quad z_{\tau}=0
\end{aligned}
$$

(where the time in $I_{\tau}$, called $t_{\tau}$, is set to zero at the instant, when $S$ and $I_{\tau}$ (locally) coincide, which happens at proper time $\tau$ ), we find

$$
\begin{aligned}
& \mathbf{S}_{*}^{\mathrm{zp}}=\frac{c}{4 \pi}\left\langle 0\left|\mathbf{E}_{\tau}^{\mathrm{zp}} \times \mathbf{B}_{\tau}^{\mathrm{zp}}\right| 0\right\rangle_{*}= \\
& =\frac{c}{4 \pi}\left\{\hat{x}\left\langle 0\left|E_{y} B_{z}-E_{z} B_{y}\right| 0\right\rangle+\hat{y} \times\right. \\
& \left.\times\left\langle 0\left|E_{z} B_{x}-E_{x} B_{z}\right| 0\right\rangle+\hat{z}\left\langle 0\left|E_{x} B_{y}-E_{y} B_{x}\right| 0\right\rangle\right\} .
\end{aligned}
$$

The star in Eq. (65) implies that the quantity needs to be evaluated in the laboratory inertial frame $I_{*}$. It turns out that only two terms of the $x$-component of the Poynting vector (65) are non-vanishing, and seven other components are zero; so, exact calculations lead to the following result:

$\mathbf{S}_{*}^{\mathrm{zp}}=-\hat{x} \frac{8 V^{3}}{3 \hbar^{2} \pi^{2} n^{3} c^{2}} \sinh \left(\frac{2 \alpha \tau}{c}\right) \int\left(\rho_{\mathrm{pE}}-\rho\right)^{3} d \rho$. 
Quantity (66) represents the energy flux, namely the energy, associated with fluctuations of the 3D quantum vacuum, which enters the uniformly accelerating object's body per unit area per unit time from the viewpoint of the observer at rest in the inertial laboratory frame $I_{*}$. The energy flux (66) generates a momentum - associated with fluctuations of the quantum vacuum energy density - the object has swept through after a time duration $t_{*}$, which, as judged again from the $I_{*}$-frame viewpoint, is

$p_{*}=g_{*} V_{*}=\frac{S_{*}}{c^{2}} V_{*}=-\hat{x} \frac{1}{6 \pi c} \gamma_{\tau}^{2} \beta_{\tau}\left\langle E_{*}^{2}+B_{*}^{2}\right\rangle V_{*}$.

In Eq. (67), $S_{*}$ represents the energy flux producing a parallel, $x$-directed momentum density, namely a field momentum growth per unit time and per unit volume, as it is incoming toward the object position, $\left(c^{2} / a, 0,0\right)$ of $S$ at the object proper time $\tau$ and as estimated from the viewpoint of $I_{*}$. In particular, if $V$ is the proper volume of the object, from the viewpoint of $I_{*}$, because of the Lorentz contraction, such volume is then $V_{*}=\frac{V}{\gamma_{\tau}}$. Thus, the amount of momentum (67) due to the field inside the volume of the object according to $I_{*}$ becomes

$p_{*}=g_{*} V_{*}=\frac{S_{*}}{c^{2}} \frac{V_{0}}{\gamma_{\tau}}=$

$=-\hat{x} \frac{4 V^{4}}{3 \hbar^{2} \pi^{2} n^{3} c^{2}} \gamma_{\tau} \beta_{\tau} \int \eta(\rho)\left(\rho_{\mathrm{pE}}-\rho\right)^{3} d \rho$.

At the proper time $\tau=0$, the $\left(c^{2} / a, 0,0\right)$ point of the laboratory inertial system $I_{*}$ instantaneously coincides and moves with the object point of the Rindler frame $S$, in which the object is fixed. Since the observer located at $x_{*}=\frac{c^{2}}{a}, y_{*}=0, z_{*}=0$ at $t_{*}=0$ coincides and moves with the object, because the latter is accelerated with a constant proper acceleration a, the object receives a time rate of change of incoming momentum - due to fluctuations of the quantum vacuum energy density - of the form:

$$
\frac{d \mathbf{p}_{*}}{d t_{*}}=\left.\frac{1}{\gamma_{\tau}} \frac{d \mathbf{p}_{*}}{d \tau}\right|_{\tau=0} .
$$

As a consequence, the radiation associated with the motions of virtual particles of the RS processes of creation/annihilation in the $3 \mathrm{D}$ quantum vacuum exerts on the object a force with respect to $I_{*}$ at $t_{*}=0$, which is given by the relation

$\mathbf{F}_{*}=\frac{d \mathbf{p}_{*}}{d t_{*}}=-\left[\frac{16 V^{4}}{3 \hbar^{2} \pi^{2} n^{3} c^{5}} \int \eta(\rho)\left(\rho_{\mathrm{pE}}-\rho\right)^{3} d \rho\right] \mathbf{a}$.

In Eq. (7), the quantity

$m_{i}=\left[4 \frac{V^{4}}{\hbar^{2} \pi^{2} n^{3} c^{5}} \int \eta(\omega)\left(\rho_{\mathrm{pE}}-\rho\right)^{3} d \omega\right]$

is an invariant scalar having the dimension of a mass, which corresponds to the fraction of the energy of the radiation - produced by the motions of virtual particles of the RS processes of creation/annihilation of quanta in the $3 \mathrm{D}$ quantum vacuum - enclosed within the object, interacting with it, and parametrized by the $\eta(\omega)$ factor in the integrand.

In this way, Sunahata's, Rueda's, and Haisch's formalism regarding the equivalence principle between inertial mass and gravitational mass based on nonrelativistic QED can receive a new reading and a new physical meaning. On one hand, we have shown in our model in a coherent unifying picture that an object accelerating through the regions of the quantum vacuum characterized by frequencies (41) of the radiation field determined by the motion of virtual particles of the RS processes experiences the resistance from the zero-point electric and magnetic fields. On the other hand, in the case of an object in a gravitational field, the electromagnetic zero-point fields of the regions of the quantum vacuum are characterized by frequencies (41) because of the radiation field determined by the motion of the virtual particles of the RS processes, which propagate on curved geodesics, accelerating with respect to the fixed object, thereby generating weight. In other words, this means that, in our approach, the equivalence principle does not need to be independently postulated, but derives directly as a consequence of the RS processes and, thus, of the elementary fluctuations of the energy density of the same 3D quantum vacuum.

\section{The Quantum behavior of Matter and Non-Locality}

The other important merit of our approach lies in the possibility to derive the fundamental equations of quantum theory. By starting from the relativistic hydrodynamical equation (37), adding the continuity 
equation, and observing that the product of the pressure due to the collisions of virtual particles and the volume of the region into consideration divided by the number of RS processes characterizing this region is the quantum potential (which is obtained applying the internal pressure to Fick's laws, in analogy to the treatment provided by Sbitnev in [22]), one can derive the fundamental quantum equations of standard quantum theory (the Klein-Gordon equation and, in the non-relativistic limit, the Schrödinger equation).

The pressure $p$ due to the collisions of virtual particles of the vacuum can be expressed as

$p=p_{1}+p_{2}$,

where

$p_{1}=-\frac{D^{2}}{c^{2}}\left[\nabla^{2} \Delta \rho_{\mathrm{qvE}}-\frac{1}{c^{2}} \frac{\partial^{2}}{\partial t^{2}} \Delta \rho_{\mathrm{qvE}}\right]$

derives from Fick's law and

$p_{2}=\frac{D^{2}}{2 \Delta \rho_{\mathrm{qvE}} c^{2}}\left[\left(\nabla \Delta \rho_{\mathrm{qvE}}\right)^{2}-\frac{1}{c^{2}}\left(\frac{\partial}{\partial t} \Delta \rho_{\mathrm{qvE}}\right)^{2}\right]$

is the pressure corresponding to the average momentum transfer per unit area per unit time. Equations (72)-(74) allow us to define the quantum potential associated with virtual particles of the RS processes of the 3D quantum vacuum:

$$
\begin{aligned}
& Q=V \frac{p_{1}+p_{2}}{n}=-\frac{D^{2}}{c^{2}}\left[\nabla^{2} \Delta \rho_{\mathrm{qvE}}-\frac{1}{c^{2}} \frac{\partial^{2}}{\partial t^{2}} \Delta \rho_{\mathrm{qvE}}\right]+ \\
& +\frac{D^{2}}{2 \Delta \rho_{\mathrm{qvE}} c^{2}}\left[\left(\nabla \Delta \rho_{\mathrm{qvE}}\right)^{2}-\frac{1}{c^{2}}\left(\frac{\partial}{\partial t} \Delta \rho_{\mathrm{qvE}}\right)^{2}\right]= \\
& =-\frac{\hbar^{2} c^{2} n}{2 \Delta \rho_{\mathrm{qvE}} V} \frac{\partial_{\mu} \partial^{\mu} R}{R}
\end{aligned}
$$

where $R$ is the square root of the density distribution of virtual particles in the vacuum (and, of course, $D$ is the diffusion coefficient given by (40)). The quantum potential (75) describes the influence through the pressures that arise between ensembles of virtual particles populating the vacuum.

Now, on the basis of Eqs. (36) and (75), we have

$$
\left(\frac{\varepsilon+p}{p} \gamma\right) \partial^{\nu} u_{\nu} u^{\nu}-\partial^{\nu}\left(\frac{\varepsilon+p}{p} \gamma\right)+\partial^{\nu} Q=0
$$

where

$\gamma=\left(1-\frac{v^{2}}{c^{2}}\right)^{-1 / 2}$.
The solution of (76) is

$\frac{\Delta \rho_{\mathrm{qvE}} V}{c^{2} n} v^{2}-\frac{\Delta \rho_{\mathrm{qvE}} V}{c^{2} n} c^{2}+2 Q=2 C$,

$C$ being an integration constant having the dimension of energy.

Here, by introducing the scalar field $S+\hbar \omega \gamma^{-1} t$, where $S$ characterizes a degree of mobility of the virtual particles in a vicinity of the 4 -point $(t, \mathbf{r})$, taking into account that the velocity can be expressed as

$$
\begin{aligned}
& v^{2}=\frac{c^{4} n^{2}}{\left(\Delta \rho_{\mathrm{qvE}}\right)^{2} V^{2}} \partial_{\mu} S \partial^{\mu} S+ \\
& +\frac{2 E c^{3} n^{2}}{\gamma\left(\Delta \rho_{\mathrm{qvE}}\right)^{2} V^{2}} \partial_{0} S+\frac{E^{2} c^{2} n^{2}}{\gamma^{2}\left(\Delta \rho_{\mathrm{qvE}}\right)^{2} V^{2}},
\end{aligned}
$$

one obtains

$$
\begin{aligned}
& \partial_{\mu} S \partial^{\mu} S+\frac{2 E}{c \gamma} \partial_{0} S+\frac{E^{2}}{c^{2} \gamma^{2}}-\frac{\left(\Delta \rho_{\mathrm{qvE}}\right)^{2} V^{2}}{c^{2} n^{2}}- \\
& -\hbar^{2} \frac{\partial_{\mu} \partial^{\mu} R}{R}=2 \frac{\left(\Delta \rho_{\mathrm{qvE}}\right) V}{c^{2} n} C .
\end{aligned}
$$

In the relativistic limit, one has $v \rightarrow c, \gamma \rightarrow \infty$, and, thus, $\frac{2 E}{c \gamma} \partial_{0} S+\frac{E^{2}}{c^{2} \gamma^{2}} \rightarrow 0$; in this regime, Eq. (80) becomes therefore

$$
\begin{aligned}
& \partial_{\mu} S \partial^{\mu} S-\frac{\left(\Delta \rho_{\mathrm{qvE}}\right)^{2} V^{2}}{c^{2} n^{2}}- \\
& -\hbar^{2} \frac{\partial_{\mu} \partial^{\mu} R}{R}=2 \frac{\left(\Delta \rho_{\mathrm{qvE}}\right) V}{c^{2} n} C,
\end{aligned}
$$

which is a quantum Hamilton-Jacobi equation extracted from the Klein-Gordon-type equation for the energy density of the quantum vacuum

$\partial_{\mu} \partial^{\mu} \psi+\frac{\left(\Delta \rho_{\mathrm{qvE}}\right)^{2} V^{2}}{\hbar^{2} c^{2} n^{2}} \psi+2 \frac{\left(\Delta \rho_{\mathrm{qvE}}\right) V}{\hbar^{2} c^{2} n} C \psi=0$,

where $\psi=R \exp (i S / \hbar)$ is the wave function of the quantum vacuum. The Klein-Gordon equation of ordinary quantum mechanics may be obtained directly as a special case of (82) by the replacement $m=\frac{\Delta \rho_{\mathrm{qvE}} V}{c^{2} n}$ for the mass of a particle.

In the non-relativistic limit, one has $v \rightarrow 0, \gamma \rightarrow 1$, and, thus, $\frac{E^{2}}{c^{2}}-\frac{\left(\Delta \rho_{\mathrm{qvE}}\right)^{2} V^{2}}{c^{2} n^{2}}=0$; in this way, after some mathematical manipulations, one obtains

$\frac{\partial}{\partial t} S+\frac{c^{2} n}{2\left(\Delta \rho_{\mathrm{qvE}_{0}}\right) V}(\nabla S)^{2}-$

ISSN 2071-0194. Ukr. J. Phys. 2016. Vol. 61, No. 5 
$-\hbar^{2} \frac{c^{2} n}{2\left(\Delta \rho_{\mathrm{qvE}_{0}}\right) V} \frac{\nabla^{2} R}{R}=C$,

where $\Delta \rho_{q v E_{0}}$ are fluctuations of the quantum vacuum energy density at rest. Equation (83) is the quantum Hamilton-Jacobi equation, which can be drawn from the Schrödinger-type equation for the energy density of quantum vacuum

$i \hbar \frac{\partial \psi}{\partial t}=-\hbar^{2} \frac{c^{2} n}{2\left(\Delta \rho_{\mathrm{qvE}_{0}}\right) V} \nabla^{2} \psi-C \psi$

for the wave function $\psi=R \exp (i S / \hbar)$ describing the quantum vacuum. Even here, the Schrödinger equation of ordinary quantum mechanics may be obtained directly as a special case of (84) by the replacement $m=\frac{\Delta \rho_{\mathrm{qvE}} V}{c^{2} n}$ for the mass of a particle.

On the basis of Eqs. (72)-(84), one can conclude that the view of the 3D quantum vacuum as a superfluid medium, which consists of the enormous amount of RS processes of creation/annihilation of virtual pairs of particles-antiparticles (thus constituting an organized Bose ensemble) and which is defined by the energy-momentum tensor (36), allows us to derive directly the quantum behavior of matter. In fact, the appropriate Hamilton-Jacobi equations - associated with Klein-Gordon and Schrödinger equations respectively - emerge as a result of the pressure due to the collisions of virtual particles of the medium, which is linked with fluctuations of the quantum vacuum energy density. In this picture, the fundamental point to emphasize and to remark is that the quantum potential (75) of the vacuum may be considered as the real origin of the quantum effects. The quantum behavior of matter, as we know it from standard quantum theory, derives ultimately just from the quantum potential, which describes the geometry of the vacuum through the pressures that arise by the collisions between virtual particles-antiparticles populating the vacuum corresponding to the RS processes.

As a consequence, the non-local correlations between the quantum particles associated with the ordinary quantum potential can be also considered as an effect of the quantum potential of the quantum vacuum $(75)$.

In a series of recent papers [36,37], Fiscaletti and Licata introduced, as a parameter of the non-local correlations (and thus that provides a direct measure of the degree of departure of a system from the Euclidean geometry characteristic of classical physics), a quantum-entropic length (called also Bell length, in honour of J.S. Bell (1928-1990)). The Bell length is

$L_{\text {quantum }}=\frac{1}{\sqrt{\left(\nabla S_{Q}\right)^{2}-\nabla^{2} S_{Q}}}$,

where the logarithmic function

$S_{Q}=-\frac{1}{2} \ln \rho$

is defined as the quantum entropy associated with the physical system. The quantum entropy (86) can be interpreted as the physical entity that, in the quantum domain, describes the degree of order and chaos of the vacuum supporting the density $\rho=|\psi(\mathbf{x}, t)|^{2}$ describing the space-temporal distribution of the ensemble of particles associated with the wave function of the physical system under consideration and allows the quantum potential of non-relativistic quantum mechanics to be expressed as

$Q=-\frac{\hbar^{2}}{2 m}\left(\nabla S_{Q}\right)^{2}+\frac{\hbar^{2}}{2 m}\left(\nabla^{2} S_{Q}\right)$,

namely as an information channel given by the sum of two quantum correctors (of the kinetic energy and of the potential energy, respectively) linked with the quantum entropy [38-40]. The maximum value of Bell length (85) is obtained for $L_{\text {quantum }}^{\max }=1$, which corresponds to the maximum delocalization of the quantum system $[36,37]$.

Now, on the basis of the approach of the 3D quantum vacuum proposed in this article, the Bell length (85) characterizing ordinary non-relativistic quantum mechanics (and based on two quantum correctors to the energy of the system) may be generalized into a more general Bell length of the 3D quantum vacuum (as the ultimate visiting card, which is responsible for the non-local correlations between elementary regions of the physical space). In fact, by comparing Eqs. (75) and (87), one may make the positions

$$
\begin{aligned}
& -\frac{\hbar^{2}}{2 m}\left(\nabla S_{Q}\right)^{2}=\frac{D^{2}}{2 \Delta \rho_{\mathrm{qvE}} c^{2}} \times \\
& \times\left[\left(\nabla \Delta \rho_{\mathrm{qvE}}\right)^{2}-\frac{1}{c^{2}}\left(\frac{\partial}{\partial t} \Delta \rho_{\mathrm{qvE}}\right)^{2}\right]
\end{aligned}
$$

for the quantum corrector to the kinetic energy and

$$
\frac{\hbar^{2}}{2 m}\left(\nabla^{2} S_{Q}\right)=-\frac{D^{2}}{c^{2}}\left[\nabla^{2} \Delta \rho_{\mathrm{qvE}}-\frac{1}{c^{2}} \frac{\partial^{2}}{\partial t^{2}} \Delta \rho_{\mathrm{qvE}}\right]
$$


for the quantum corrector to the potential energy. Therefore, one can suggest that the term $\frac{D^{2}}{2 \Delta \rho_{\mathrm{qvE}} c^{2}} \times \times\left[\left(\nabla \Delta \rho_{\mathrm{qvE}}\right)^{2}-\frac{1}{c^{2}}\left(\frac{\partial}{\partial t} \Delta \rho_{\mathrm{qvE}}\right)^{2}\right]$ origins the quantum corrector to the kinetic energy, and that the term $-\frac{D^{2}}{c^{2}}\left[\nabla^{2} \Delta \rho_{\mathrm{qvE}}-\frac{1}{c^{2}} \frac{\partial^{2}}{\partial t^{2}} \Delta \rho_{\mathrm{qvE}}\right]$ origins the quantum corrector to the potential energy of the physical system into consideration. In other words, in our approach, the quantum correctors to the energy of the system derive from more fundamental entities linked with elementary fluctuations of the energy density of the 3D quantum vacuum. On the basis of Eqs. (88) and (89), one obtains

$\left(\nabla S_{Q}\right)^{2}=\frac{D^{2} V}{c^{4} \hbar^{2} n}\left[-\left(\nabla \Delta \rho_{\mathrm{qvE}}\right)^{2}+\frac{1}{c^{2}}\left(\frac{\partial}{\partial t} \Delta \rho_{\mathrm{qvE}}\right)^{2}\right]($

and

$\left(\nabla^{2} S_{Q}\right)=-\frac{2 D^{2} \Delta \rho_{\mathrm{qvE}} V}{c^{4} \hbar^{2} n}\left[\nabla^{2} \Delta \rho_{\mathrm{qvE}}-\frac{1}{c^{2}} \frac{\partial^{2}}{\partial t^{2}} \Delta \rho_{\mathrm{qvE}}\right]$,

respectively. Thus, the Bell length (85) of ordinary non-relativistic quantum mechanics derives from the more general Bell length of the 3D quantum vacuum

$$
\begin{aligned}
& L_{\text {quantum }}= \\
& =\left(c^{2} \hbar\right) /\left\{D \left(\frac { V } { n } \left[-\left(\nabla \Delta \rho_{\mathrm{qvE}}\right)^{2}+\frac{1}{c^{2}}\left(\frac{\partial}{\partial t} \Delta \rho_{\mathrm{qvE}}\right)^{2}-\right.\right.\right. \\
& \left.\left.\left.-\Delta \rho_{\mathrm{qvE}}\left(\nabla^{2} \Delta \rho_{\mathrm{qvE}}-\frac{1}{c^{2}} \frac{\partial^{2}}{\partial t^{2}} \Delta \rho_{\mathrm{qvE}}\right)\right]\right)^{1 / 2}\right\} .
\end{aligned}
$$

The quantum length (92) can be considered as the ultimate visiting card, which is responsible for the nonlocal correlations in the quantum domain. The quantum length (92) is the ultimate parameter indicating that, at a fundamental level, the $3 \mathrm{D}$ quantum vacuum defined by RS processes of creation/annihilation of virtual particles-antiparticles organized in Bose ensembles and corresponding to fluctuations of the quantum vacuum energy density is a non-local timeless manifold.

The maximum value of the Bell length of the 3D quantum vacuum (92), which implies the maximum delocalization of a quantum system, is 1 , which means

$\left(c^{2} \hbar\right) /\left\{D\left(\frac{V}{n}\left[-\left(\nabla \Delta \rho_{\mathrm{qvE}}\right)^{2}+\frac{1}{c^{2}}\left(\frac{\partial}{\partial t} \Delta \rho_{\mathrm{qvE}}\right)^{2}-\right.\right.\right.$
$\left.\left.\left.-\Delta \rho_{\mathrm{qvE}}\left(\nabla^{2} \Delta \rho_{\mathrm{qvE}}-\frac{1}{c^{2}} \frac{\partial^{2}}{\partial t^{2}} \Delta \rho_{\mathrm{qvE}}\right)\right]\right)^{1 / 2}\right\}=1$,

namely,

$\left(2 \Delta \rho_{\mathrm{qvE}} V\right) /\left\{n\left(\frac{V}{n}\left[-\left(\nabla \Delta \rho_{\mathrm{qvE}}\right)^{2}+\frac{1}{c^{2}}\left(\frac{\partial}{\partial t} \Delta \rho_{\mathrm{qvE}}\right)^{2}-\right.\right.\right.$

$\left.\left.\left.-\Delta \rho_{\mathrm{qvE}}\left(\nabla^{2} \Delta \rho_{\mathrm{qvE}}-\frac{1}{c^{2}} \frac{\partial^{2}}{\partial t^{2}} \Delta \rho_{\mathrm{qvE}}\right)\right]\right)^{1 / 2}\right\}=1$,

namely,

$\left(2 \Delta \rho_{\mathrm{qvE}} V^{1 / 2}\right) /\left\{n^{1 / 2}\left(\left[-\left(\nabla \Delta \rho_{\mathrm{qvE}}\right)^{2}+\frac{1}{c^{2}}\left(\frac{\partial}{\partial t} \Delta \rho_{\mathrm{qvE}}\right)^{2}-\right.\right.\right.$

$\left.\left.\left.-\Delta \rho_{\mathrm{qvE}}\left(\nabla^{2} \Delta \rho_{\mathrm{qvE}}-\frac{1}{c^{2}} \frac{\partial^{2}}{\partial t^{2}} \Delta \rho_{\mathrm{qvE}}\right)\right]\right)^{1 / 2}\right\}=1$.

In this way, one obtains a simple relation satisfied by the number of virtual particles-antiparticles of the RS processes of the 3D quantum vacuum under the condition of maximum entanglement and delocalization in a quantum system:

$$
\begin{aligned}
& n^{1 / 2}=\left(2 \Delta \rho_{\mathrm{qvE}} V^{1 / 2}\right) / \\
& /\left\{\left(\left[-\left(\nabla \Delta \rho_{\mathrm{qvE}}\right)^{2}+\frac{1}{c^{2}}\left(\frac{\partial}{\partial t} \Delta \rho_{\mathrm{qvE}}\right)^{2}-\right.\right.\right. \\
& \left.\left.\left.-\Delta \rho_{\mathrm{qvE}}\left(\nabla^{2} \Delta \rho_{\mathrm{qvE}}-\frac{1}{c^{2}} \frac{\partial^{2}}{\partial t^{2}} \Delta \rho_{\mathrm{qvE}}\right)\right]\right)^{1 / 2}\right\} .
\end{aligned}
$$

Equation (96) indicates a condition, which must be satisfied by the number of RS processes in a given volume of the physical space in order to give origin to the maximum grade of non-locality in a quantum system having the mass $m=\frac{\Delta \rho_{\mathrm{qvE}} V}{c^{2} n}$ produced by fluctuations of the quantum vacuum energy density corresponding to the same RS processes.

\section{Conclusions}

A 3D quantum vacuum intended as a superfluid medium consisting of an enormous amount of RS processes of creation/annihilation of particlesantiparticles with opposite orientations of spins is the fundamental background, which determines a unifying view of gravity, electromagnetic fields, and quantum behavior of matter as different aspects of the same fluctuations of the quantum vacuum energy density. In the 3D quantum vacuum model, general relativity emerges as the hydrodynamic limit of the underlying geometry of the microscopic 3D quantum vacuum condensate. The curvature of space-time characteristic of general relativity may be considered

ISSN 2071-0194. Ukr. J. Phys. 2016. Vol. 61, No. 5 
as a mathematical value, which emerges from the quantized metric of the $3 \mathrm{D}$ quantum vacuum linked with fluctuations of the quantum vacuum energy density, and the dark energy is itself a consequence of specific fluctuations of the quantum vacuum energy density.

As a consequence of the evolution of the RS processes, namely of the motion of virtual particlesantiparticles generated in the RS processes, the spacetime is filled with a virtual radiation with frequencies depending on the fluctuations of the quantum vacuum energy density. The frequency of this virtual radiation may be considered as the origin of the electromagnetic effects of the $3 \mathrm{D}$ quantum vacuum. In particular, the electromagnetic field inside a perfectly reflecting cavity is associated with the infinite different modes of the fundamental 3D quantum vacuum, where each mode corresponds to an independent oscillation defined by the frequency produced by a specific RS process of creation/annihilation of quanta in correspondence to elementary fluctuations of the 3D quantum vacuum. In this approach, the inertial mass of the object emerges from the interacting fraction of an energy density characterizing the electromagnetic properties of the 3D quantum vacuum, which are determined by the frequencies associated to opportune RS processes of creation/annihilation corresponding to elementary fluctuations of the quantum vacuum energy density. As a consequence, the explanation of the weak equivalence principle provided by Haisch, Rueda, and Puthoff gets a new simple, suggestive, and more unifying re-reading: here, the equivalence principle does not need to be independently postulated, but derives directly as a consequence of the RS processes and, thus, of elementary fluctuations of the energy density of the same 3D quantum vacuum.

Finally, the view of the 3D quantum vacuum allows us to derive directly the quantum behavior of matter, yielding appropriate Hamilton-Jacobi equations - associated with Klein-Gordon and Schrödinger equations, respectively - as a result of the pressure due to the collisions of virtual particles of the medium, which is linked with fluctuations of the quantum vacuum energy density. In this picture, the quantum behavior of matter, as we know it from standard quantum theory, derives ultimately just from a quantum potential of the $3 \mathrm{D}$ quantum vacuum, which describes the geometry of the vacuum through the pressures that arise between the collisions of virtual particles- antiparticles populating the vacuum corresponding to the RS processes. The quantum potential of the $3 \mathrm{D}$ quantum vacuum leads to the definition of a Bell length as the ultimate parameter, which measures the degree of non-local correlation in the quantum domain. Thus, it indicates that, at a fundamental level, the $3 \mathrm{D}$ quantum vacuum defined by RS processes of creation/annihilation of virtual particles-antiparticles organized in Bose ensembles and corresponding to fluctuations of the quantum vacuum energy density is a non-local timeless manifold.

The analysis made in this paper allows us to conclude with some considerations of epistemological character. The hypothesis that the observed world is a manifestation of a deeper dimension, besides being a basic element of the Indian philosophy, as well as of the Upanishads, has been updated in contemporary physics. A concept of this kind was already enunciated by David Bohm: "What we experience through the senses as empty space is the ground for the existence of everything, including ourselves. The things that appear to our senses are derivative forms and their true meaning can be seen only when we consider the plenum, in which they are generated and sustained, and into which they must ultimately vanish".

At the leading edge of science, the space is rediscovered as a fundamental physical reality. It is the fundamental matrix, from which the universe arises, in and through which it evolves, and into which it again re-descends. The paradigm of the $3 \mathrm{D}$ timeless non-local quantum vacuum proposed in this paper maintains that the timeless non-local quantum vacuum is the holographic matrix of the universe.

On the other hand, the notion of a holographic universe surfaced recently when a Fermilab physicist Craig Hogan suggested in the spring of 2013 that the fluctuations observed by the gravity-wave detector GEO600 may be owed to the granular nature of the spacetime at the most fundamental level, on the Planck scale, and, thus, to the most minuscule ripples or blocks of the universe [41]. The gravity wavedetector did find inhomogeneities in the matrix that constitutes the spacetime, but they were not gravitywaves. They could, however, be the elementary RS processes of creation/annihilation of quanta on the Planck scale, implied by the approach of the 3D timeless non-local quantum vacuum.

The holographic matrix that underlies things and events in the spacetime can be rightfully associ- 
ated with the primordial 3D timeless non-local quantum vacuum. The primordial non-local quantum vacuum is the universal gravitational field that produces the gravitational interaction between material objects; it is the origin of the fundamental electromagnetic fields that convey electric and magnetic effects through space; it is the ensemble of the quantum fields that vehicle the behavior of quanta; and it is the fundamental manifold that creates non-local correlations among quanta. It is the integration of these elements that gives a dimension that is beyond the spacetime but that acts on, or "in-forms," the spacetime.

According to the paradigm of the 3D timeless nonlocal quantum vacuum, the cosmos may be seen as an integral system actualizing in the interaction of two dimensions or different levels of physical reality: an unobservable deep dimension, and an observable manifest dimension. On the basis of the treatment made in this paper, the deep dimension is just the $3 \mathrm{D}$ timeless non-local quantum vacuum. The possibility is opened that the $3 \mathrm{D}$ timeless non-local quantum vacuum and the manifest dimension interact. Events in the manifest dimension structurize the $3 \mathrm{D}$ timeless non-local quantum vacuum: they alter its potential to act - to "in-form" - the manifest dimension. The 3 D timeless non-local quantum vacuum "in-forms" the manifest dimension, and the informed manifest dimension acts on - "de-forms" - the 3D timeless nonlocal quantum vacuum. The manifest dimension and the 3D timeless non-local quantum vacuum do not signify a cosmos split in two. The cosmos is one, but for the observer it is meaningfully considered under the heading of two dimensions: a fundamental dimension and an experienced dimension. The diversity of events in the experienced dimension is a manifestation of the unity that governs their interaction in the fundamental dimension. This can be considered as the basic tenet of the cosmology prospected by the view of the $3 \mathrm{D}$ timeless non-local quantum vacuum.

1. D. Fiscaletti and A. Sorli, Foundations of Physics 45, 105 (2015).

2. D. Fiscaletti and A. Sorli, Found. of Sci. 20, 387 (2015).

3. P.W. Milonni, The Quantum Vacuum - an Introduction to Quantum Electrodynamics (Academic Press, New York, 1994).

4. J.W.M. Bush, Ann. Rev. of Fluid Mech. 47, 1, 269 (2015).
5. E. Santos, Quantum vacuum fluctuations and dark energy, arXiv:0812.4121v2 [gr-qc] (2009).

6. E. Santos, Int. J. of Theor. Phys. 50, 7, 2125 (2010).

7. E. Santos, Dark energy as a curvature of space-time induced by quantum vacuum fluctuations, arXiv:1006.5543 (2010).

8. P.I. Fomin and A.P. Fomina, Problems of Atomic Science and Technology 3, 85, 10 (2013).

9. D.C. Daywitt, Progr. in Phys. 1, 27 (2009).

10. D. Fiscaletti and A. Sorli, Ann. UMCS Sectio AAA: Physica LXIX, 55 (2014).

11. D. Fiscaletti and A. Sorli, SOP Trans. on Theor. Phys. 1, 3, 11 (2014).

12. B. Greene, The Elegant Universe (Vintage Books. A Division of Random House, New York, 1999).

13. A. Sorli, J. of Adv. Phys. 1, 1, 110 (2012).

14. S. Ghao, Why gravity is fundamental, arXiv: 1001-3029v3 (2010).

15. Y.J. Ng, Phys. Lett. B 657, 1, 10 (2007).

16. Y.J. Ng, Entropy 10, 441 (2008).

17. Y.J. Ng, Holographic quantum foam, arXiv: 1001.0411v1 [gr-qc] (2010).

18. Y.J. Ng, Various facets of spacetime foam, arXiv: 1102.4109.v1 [gr-qc] (2011).

19. L. Chiatti, in: Space-Time Geometry and Quantum Events, edited by I. Licata (Nova Sci., New York, 2014), p. 11; e-print arXiv.org/pdf/1204.6636 (2012).

20. I. Licata, Eur. Phys. J. Web of Conf. (2013).

21. V. Sbitnev, From the Newton's laws to motion of the fluid and superfluid vacuum: vortex tubes, rings, and others, arXiv: 1403.3900v2 [physics.flu-dyn] (2014).

22. V. Sbitnev, Hydrodynamics of the physical vacuum: dark matter is an illusion, arXiv: 1507.03519v1 [physics.gen-ph] (2015).

23. V.I. Sbitnev, in: Selected Topics in Applications of Quantum Mechanics, edited by M.R. Pahlavani(InTech, Rijeka 2015), pp. 345; doi: 10.5772/59040.

24. V.I. Sbitnev, Found. of Phys., in press, 2015; http://arxiv.org/abs/1504.07497.

25. G.E. Volovik, The Universe in a Helium Droplet (Clarendon Press, Oxford, 2003).

26. E. Nelson, Phys. Rev. 150, 1079 (1966); doi: 10.1103/PhysRev.150.1079.

27. V.I. Sbitnev, in: Advances in Quantum Mechanics, edited by P. Bracken (InTech, Rijeka, 2013), p. 183; doi: $10.5772 / 53471$.

28. B. Haisch, A. Rueda, and H.E. Puthoff, Phys. Rev. A 49, 678 (1994).

29. B. Haisch, A. Rueda, and H.E. Puthoff, Specul. in Sci. and Techn. 20, 99 (1997).

30. A. Rueda and B. Haisch, Found. of Phys. 28, 1057 (1998).

31. A. Rueda and B. Haisch, Phys. Lett. A 240, 115 (1998).

ISSN 2071-0194. Ukr. J. Phys. 2016. Vol. 61, No. 5 
32. B. Haisch, A. Rueda, and Y. Dobyns, Ann. der Phys. 10, 393 (2001).

33. A. Rueda and B. Haisch, Ann. der Phys. 14, 479 (2005); e-print arXiv: gr-qc0504061v3 (2005).

34. H. Sunahata, A. Rueda, and B. Haisch, Quantum vacuum and inertial reaction in non-relativistic QED, arXiv: 1306.6036v1 [physics.gen-ph] (2013).

35. Y.S. Levin, Phys. Rev. A 79, 012114 (2009).

36. I. Licata and D. Fiscaletti, Axioms 3, 153 (2014).

37. D. Fiscaletti and I. Licata, Int. J. of Theor. Phys. 54, 2362 (2015).

38. D. Fiscaletti, Ukr. J. Phys. 57, 946 (2012).

39. D. Fiscaletti, Quantum Matter 2, 45 (2013).

40. D. Fiscaletti, Quantum Matter 3, 177 (2014).

41. C. Hogan, Measurement of quantum geometry using laser interferometry, arXiv: 1307.4676v1 [gr-qc] (2013).

Received 07.10.15
Д. Фіскалетті, А. Сорлі

ПРО ТРИВИМІРНИЙ КВАНТОВИЙ

ВАКУУМ ЯК ОСНОВНУ ПРИЧИНУ ГРАВІТАЦЇ, ЕЛЕКТРОМАГНІТНОГО ПОЛЯ, ТЕМНОЇ ЕНЕРГІЇ ... I КВАНТОВОЇ ПОВЕДІНКИ

\section{$\mathrm{P}$ е $з$ ю м е}

Запропоновано модель об'єднання, в якій матерія - енергетична структура тривимірного квантового вакууму, що зменшує його щільність енергіі. Маса і гравітація створюються змінною щільністю енергії тривимірного квантового вакууму. Електричне і магнітне поля переносяться областями поляризованого квантового вакууму з відповідними коливаннями, залежними від флуктуацій щільності енергії квантового вакууму. Квантова поведінка матерії випливає безпосередньо 3 простих флуктуацій енергії тривимірного квантового вакууму. Темна енергія - це сама енергія тривимірного квантового вакууму. 